flussungsmöglichkeit für betriebliche Kosten- und Umweltentlastungen. Da die Herbizide unter den Pflanzenschutzmitteln einen beachtlichen Mengenanteil von rund 75 Prozent einnehmen, ist beispielsweise zu prüfen, ob einzelne Herbizide wirklich erforderlich sind oder ob nicht mit alternativen Maßnahmen der Entstehung der Unkräuter vorgebeugt bzw. deren Beseitigung umwelt- und kostenschonender durchgeführt werden kann.

Weitere Ansatzpunkte werden in der Anpassung der Fruchtfolgeauswahl und in der Optimierung der Verfahren bei der Ausbringung der Dünger und Pflanzenschutzmittel gesehen. Zwei weitere Verbesserungsvorschläge betreffen die interne Verwertung des in der Agrargenossenschaft kostenneutral anfallenden Holzes und eventuell auch

\section{Integrierte Technik integrieren?}

\section{Seit über 25 Jahren werden in Deutschland Umweltbelastungen und Umwelt- schutzkosten statistisch erfasst. Letzteres ist allerdings methodisch nicht un- problematisch. Deshalb werden seit fünf Jahren nur noch End-of-Pipe-Umwelt- schutzinvestitionen erhoben. Die EU möchte dies nun allerdings ändern.}

$\mathrm{U}$ Von Bernd Becker mweltstatistiken basieren auf Gesetzen, die von Zeit zu Zeit an neue Anforderungen angepasst werden. Das deutsche Umweltstatistikgesetz von 1994 hat im Vergleich zur Fassung von 1974 durch die Einführung mehrerer zusätzlicher und die Modifizierung bestehender Erhebungen das umweltpolitische Informationsangebot verbessert. Gleichzeitig war man 1994 bestrebt, die Betriebe der gewerblichen Wirtschaft zu entlasten, indem man vor allem die statistische Nutzung von umweltrelevanten Verwaltungsunterlagen ermöglichte.

Im Rahmen des jetzigen Umweltstatistikgesetzes werden zwei Typen von umweltpolitischen Merkmalen erhoben:

- Feststellung der Umweltbelastungen durch Emissionen, und zwar in den Bereichen Abfall, Wasser und Luft.

- Quantifizierung der ökonomischen Bedeutung des Umweltschutzes.

Insgesamt werden in den Bereichen Abfallwirtschaft, Wasserwirtschaft, Luftreinhaltung und Umweltökonomie mehr als 30 umweltstatistische Erhebungen durchgeführt (vgl. Tab. 1), die im Folgenden zunächst näher erläutert werden.

Wohl der Allgemeinheit und im Einklang mit ihm auch dem Nutzen Einzelner dienen und dass jede vermeidbare Beeinträchtigung unterbleibt. Wohl aus diesen Gründen sind die Statistiken der Wasserwirtschaft die ältesten der hier betrachteten. Nach heutigem Stand umfasst das Umweltstatistik-
Strohs zur Energieerzeugung in einem dezentralen Biomasseheizkraftwerk und den Einsatz verarbeiteten, selbst produzierten Rapses als Biodiesel für den betriebseigenen Fuhrpark.

Für eine systematische und kontinuierliche Identifizierung und Prüfung derartiger ökonomischökologischer Optimierungsmaßnahmen wird für die Agrargenossenschaft momentan ein unternehmensspezifisches SEFR-System entwickelt. Die Basis bildet eine Excel-Tabellenkalkulation, die bei Bedarf Ausgangspunkt für eine Access-Datenbank sein kann. Die Microsoft-Produktpalette ist - wie in vielen kleinen und mittelständischen Unternehmen - in der Agrargenossenschaft vorhanden und erfordert folglich geringen Einarbeitungsaufwand.

\section{Anmerkungen}

(1) Basis hierfür war eine systematische Vorstudie. Vgl. Kramer, M./ Brauweiler, J./ Lehmann, M.: Branchenstrukturanalyse: Deutschland, Polen, Tschechien und Euroregion Neisse. In: Osteuropa-Wirtschaft, Heft 3/2000, S. 221-256.

(2) Vgl. Kreeb, M.: Umweltkostenmanagement für mittelständische Unternehmen. Diskussionspapier-Heft 23, Universität Witten/Herdecke 1999.

\section{Die Autorlnnen}

Prof. Dr. Matthias Kramer ist Leiter des Studiengangs Betriebswirtschaftslehre im Internationalen Hochschulinstitut Zittau. Hans-Christian Brauweiler und Peggy Eifler sind dort wiss. Mitarbeiterlnnen.

Kontakt: IHI Zittau, Markt 23, 02763 Zittau,

Tel. 03583/ 771-531, Fax -535, eifler@ihi-zittau.de, kramer@ihi-zittau.de,brauweiler_c@ihi-zittau.de

gesetz im Bereich der Wasserwirtschaft 12 Erhebungen, die in fünf Bereiche zusammengefasst werden können (siehe Tab. 1).

\section{Luftverunreinigungen}

Die Erhebungen zur Luftreinhaltung wurden mit dem Umweltstatistikgesetz von 1994 neu eingeführt. Im einzelnen sind dies zwei Erhebungen, nämlich die Erhebung der Luftverunreinigungen und die Erhebung bestimmter ozonschichtschädigender und klimawirksamer Stoffe.

Bei der Erhebung der Luftverunreinigungen wird darauf verzichtet, die betroffenen Betriebe direkt zu befragen. Stattdessen werden die Emissionserklärungen ausgewertet, die von den Anlagenbetreibern an die Gewerbeaufsichtsämter bzw. Landesumweltämter übermittelt wurden.

Die Erhebung bestimmter ozonschichtschädigender und klimawirksamer Stoffe befragt Unternehmen, die entweder bestimmte ozonschichtschädigende Stoffe herstellen, einführen oder ausführen oder ozonschichtschädigende und klimawirksame Stoffe in nennenswertem Umfang zur Herstellung, Instandhaltung oder Reinigung von Erzeugnissen verwenden.

Diese Erhebung dient dazu, quantitativ festzustellen, ob die Produktion und Verwendung der ozonschichtschädigenden Stoffe in der Bundesrepublik abnimmt bzw. völlig eingestellt wird, sowie der Beobachtung der Entwicklung und des Einsatzes von klimawirksamen Ersatzstoffen.

\section{Umweltökonomische Erhebungen}

Im Bereich der umweltökonomischen Statistiken soll die ökonomische Bedeutung des Umweltschutzes quantifiziert werden. Hier sollen 
zum einen die Aufwendungen ermittelt werden, die der gewerblichen Wirtschaft durch die Vermeidung, Verringerung oder Beseitigung von Emissionen entstehen und

zum anderen die volkswirtschaftliche Bedeutung des Marktes für Umweltschutzgüter und Umweltschutzdienstleistungen dargestellt werden.

Das neue Gesetz umfaßt bezüglich der Umweltökonomie vier verschiedene Erhebungen. Mit den Erhebungen der Investitionen und der laufenden Aufwendungen für den Umweltschutz soll die Belastung der gewerblichen Wirtschaft durch die Umweltschutzauflagen festgestellt werden. Mit der Erhebung der Waren und Dienstleistungen für den Umweltschutz sowie der Erhebung der Zusammensetzung der Investitionen für den Umweltschutz wird dagegen der Umfang des Umweltschutzmarktes erfasst.

\section{Additiv versus integriert}

Die Erhebung der Investitionen für den Umweltschutz wird seit 1975 durchgeführt. Zu den Umweltschutz-Investitionen zählen alle Zugänge an Sachanlagen, deren ausschließlicher oder überwiegender Zweck der Schutz vor schädlichen Einflüssen der Produktionstätigkeit auf die Umwelt ist. Dies können Sachanlagen zum Schutz vor schädlichen Umwelteinflüssen sein, die bei der Produktionstätigkeit entstehen (produktionsbezogene Maßnahmen), oder Investitionen zur Herstellung von Erzeugnissen, die bei Ge- oder Verbrauch eine geringere Umweltbelastung hervor-rufen (produktbezogene Maßnahmen). Bei den produktionsbezogenen Maßnahmen gibt es zwei typische Fälle, nämlich die sogenannten additiven (oder vor-/ nachgeschalteten, End-of-pipe) Investitionen und die integrierten (oder vorsorgenden) Investitionen (oder clean technologies).

Im einfachen Fall der additiven Maßnahmen handelt es sich um separate, vom übrigen Produktionsprozess getrennte Anlagen, welche zum Beispiel

der Entsorgung von Abfällen (Beispiel Verbrennungsanlage),

dem Schutz von Gewässern (Beispiel Kläranlage),

der Lärmbekämpfung (Beispiel Lärmschutzwand) oder

der Luftreinhaltung (Beispiel Abluftfilter) dienen. Sie sind eindeutig als Umweltschutzmaßnahmen zu identifizieren und sind in der Regel buchhalterisch und damit auch statistisch einfach nachzuweisen.

Die integrierten Maßnahmen dagegen sind definitionsgemäß immer ein integrierter, das heißt nicht klar isolierbarer Teil einer größeren Anlage. Als Beispiele seien hier

die Kreislaufführung von Stoffen und Kühlwasser oder

- die Nutzung von Reaktionswärme (Wärmetauscher, Kopplung mit anderen Prozessen) genannt.
Tabelle 1: Erhebungen der Umweltstatistik

\begin{tabular}{|c|c|}
\hline Bereich & Erhebungsgegenstände \\
\hline \multirow[t]{6}{*}{ Abfallwirtschaft } & - Abfallentsorgung bei den Betreibern von zulassungsbedürftigen Anlagen, \\
\hline & - Abfalleinsammlung, deren Beförderung und Verbleib, \\
\hline & - besonders überwachungsbedürftige Abfälle durch sekundärstatistische Auswertungen von \\
\hline & Begleitscheinen, \\
\hline & - Entsorgung bestimmter Abfälle (wie z.B. Bauschutt, Bodenaushub, Altöl, Kunststoff, \\
\hline & Altglas und Verpackungen). \\
\hline \multirow[t]{7}{*}{ Wasserwirtschaft } & - öffentliche Wasserversorgung und Abwasserbeseitigung, \\
\hline & - Wasserversorgung und Abwasserbeseitigung im Bergbau, bei der Gewinnung von \\
\hline & Steinen und Erden sowie im Verarbeitenden Gewerbe, \\
\hline & - Wasserversorgung und Abwasserbeseitigung in der Landwirtschaft, \\
\hline & - Wasserversorgung und Abwasserbeseitigung bei Wärmekraftwerken für die öffentliche \\
\hline & Versorgung sowie \\
\hline & - Erhebungen über wassergefährdende Stoffe. \\
\hline \multirow[t]{2}{*}{ Luftreinhaltung } & - Luftverunreinigungen, \\
\hline & - bestimmte ozonschichtschädigende und klimawirksame Stoffe. \\
\hline \multirow[t]{4}{*}{ Umweltökonomie } & - Investitionen für den Umweltschutz, \\
\hline & - laufende Aufwendungen für den Umweltschutz, \\
\hline & - Waren und Dienstleistungen für den Umweltschutz, \\
\hline & - Zusammensetzung der Investitionen für den Umweltschutz \\
\hline
\end{tabular}

Integrierte Anlagen können in buchhalterischer und vor allem in statistischer Hinsicht nur schwer nachgewiesen werden. Aus Gründen der Klarheit und zur Entlastung der Unternehmen werden in dieser Erhebung ab 1996 nur die additiven Umweltschutz-Investitionen nachgewiesen.

\section{Weiterentwicklungen auf EU-Ebene}

Auf der Ebene der Europäischen Union (EU) werden mehr und mehr umweltpolitische Initiativen entwickelt, die zunehmend auch die deutsche Umweltstatistik beeinflussen. Als Beispiele seien genannt:

die Verordnung zur Statistik über die Abfallbewirtschaftung

die Änderung der Verordnung über die strukturelle Unternehmensstatistik und

- die Wasserrahmenrichtlinie.

Hinsichtlich der betrieblichen Ermittlung der Umweltschutzkosten ist die geplante Änderung der Verordnung über die strukturelle Unternehmensstatistik relevant. Hier werden zwei neue Umweltvariablen vorgeschlagen, für die Daten an die EU zu liefern sind. Dabei handelt es sich um die integrierten Umweltschutz-Investitionen und um die laufenden Aufwendungen für den Umweltschutz. Wie weiter oben ausführlich erläutert wurde, ist der statistische Nachweis der integrierten Umwelttechnik sehr schwierig. Deswegen war es nicht überraschend, dass mehrere Mitgliedstaaten auf diese Schwierigkeit hinwiesen und die neuen Umweltvariablen ablehnten, während andere die hohe politische Bedeutung dieser Daten betonten. Als Kompromiss scheint sich abzuzeichnen, dass die Datenlieferungen erst dann von den Mitgliedstaaten gefordert werden, wenn eine zur Zeit sich noch in der Vorbereitung befindende Kommissionsverordnung über die statistischen Definitionen und Operationalisierungen der neuen Umweltvariablen fertiggestellt ist.

\section{Der Autor}

Dr. Bernd Becker ist Leiter der Gruppe "Umwelt" des Statistischen Bundesamtes.

Kontakt: Statistisches Bundesamt, Gruppe VIIIB "Umwelt", Zweigstelle Bonn, Graurheindorfer Strasse 198, 53117 Bonn, Tel. 01888-644-8200, Fax -8963/-8990, E-mail: bernd.becker@destatis.de 
(c) 20I0 Authors; licensee IÖW and oekom verlag. This is an article distributed under the terms of the Creative Commons Attribution Non-Commercial No Derivates License (http://creativecommons.org/licenses/by-nc-nd/3.o/), which permits unrestricted use, distribution, and reproduction in any medium, provided the original work is properly cited. 